\title{
AÇÃO EDUCATIVA À GESTANTE FUNDAMENTADA NA PROMOÇÃO DA SAÚDE: UMA REFLEXÃO
}

Educative action for the pregnant women based upon health promotion: A reflection

Acción educativa a la mujer embarazada basada en la promoción de la salud: Una reflexión

Isolda Pereira da Silveira

Maria Grasiela Teixeira Barroso
Antônia do Carmo Soares Campos

Neiva Francinely Cunha Vieira
Francisca Ana Martins Carvalho

\section{Resumo}

Reflexão sobre a atenção à mulher no período gravídico tomando como referência as Conferências Internacionais da Promoção da Saúde e vivência profissional das autoras nesta área. Objetiva-se refletir sobre a complexidade que envolve as estratégias de Educação em Saúde destinadas à promoção da saúde da gestante, especialmente aquelas que envolvem a ação educativa transformadora por parte dos profissionais na busca da promoção da saúde.

Palavras-chave: Educação em Saúde. Mulheres Grávidas. Promoção da Saúde. Educação. Enfermagem Obstétrica.

\section{Abstract}

It's a reflection about the attention to the woman during pregnancy taking as a reference the Health Promotion International Conferences and the authors' professional experience in this area. One aims to reflect about the complexity that involves the Education and Health strategies destined for the pregnant's health, especially those that involve transforming educational action on the part of the professionals in the search of health promotion.

Keywords:

Health Education. Pregnant Women. Health Promotion. Education. Obstetrical Nursing.

\section{Resumen}

Reflexión sobre la atención a la mujer en el período de la gestación teniendo como referencial las Conferencias Internacionales de la Promoción de la Salud y vivencias profesionales de las autoras en esta área. Se objetiva reflexionar sobre la complejidad que abarca a las estrategias de Educación en Salud destinadas a la promoción de la salud de la mujer que está embarazada, especialmente aquellas que abarcan la acción educativa en transformación por parte de los profesionales en la busca de la promoción de la salud.

Palabras clave:

Educación en Salud. Mujeres Embarazadas. Promoción de la Salud. Educación. Enfermería Obstétrica. 


\section{INTRODUÇÃO}

\section{A situação atual da saúde da gestante}

A mulher necessita de informações e conhecimentos sobre a saúde reprodutiva e seus direitos para desenvolver sua gestação sadia. Para isto, a contribuição das ações públicas e programas que favoreçam o acesso aos serviços de saúde permitem melhorias na qualidade da assistência no período gravídico.

Constatou-se que o Brasil| encontra-se em situação nada confortável, com alta taxa de mortalidade materna, estimada em 73,1 por 100 nascidos vivos em 2002. Entretanto, a subnotificação é evidente, além de se constatar um mau preenchimento das declarações de óbitos maternos, observando a investigação de todos os óbitos femininos em idade fértil. Segundo pesquisadores como Costa, Ribas, Amorim e Santos; em vários casos, os registros de óbitos não mencionam a presença de gravidez, e o diagnóstico de causa básica da morte pode ocultar a causa real, associada à reprodução.

A nossa realidade é que a redução da mortalidade materna em $50 \%$ até 0 ano 2000 não foi alcançada. Essa meta foi reenfatizada pela Conferência Internacional de População e Desenvolvimento, ocorrida no Cairo em 1994, e na IV Conferência Mundial sobre a Mulher, realizada em Pequim em 1995, segundo a Rede Feminista de Saúde3.

As mortes maternas no Brasil correspondem acerca de $6 \%$ dos óbitos de mulheres de 10 a 49 anos. A maior taxa de mortalidade materna encontra-se na região Norte, seguida das regiões Centro-Oeste e Nordeste, depois a região Sul e por último a região Sudeste (Rede Feminista de Saúde ${ }^{3}$ ). Investigações sobre mortalidade materna têm mostrado ser um dado valioso no que diz respeito às condições socioeconômicas e culturais da população, bem como às condições de saúde ofertadas a ela.

Com base nos dados do ano 2001, a taxa de mortalidade materna do Estado do Ceará foi calculada em 76/ $100.000^{4}$. Antes disto, em 1994, foi constituído o Comitê Estadual de Prevenção à Mortalidade Materna, para acompanhar e recomendar providências que devem ser tomadas para a redução da taxa de mortalidade em 1994.

Nesse sentido, gestores de saúde questionam sobre 0 modelo de assistência obstétrica e perinatal que vem sendo desenvolvido nas instituições de saúde 4 . Isso porque é prioridade das políticas do Ministério da Saúde e melhorar a qualidade de vida da mulher fazendo valer sua cidadania.

Ante o exposto, o presente estudo objetiva refletir sobre a complexidade que envolve as estratégias de Educação em Saúde destinadas à promoção da saúde da gestante, especialmente aquelas que envolvem a ação educativa transformadora por parte dos profissionais na busca da promoção da saúde. Selecionamos para o desenvolvimento desta análise os seguintes eventos: Conferência Internacional sobre Cuidados Primários de Saúde realizada em Alma-Ata - Rússia, de 6 a 12 de setembro de 1978; Primeira Conferência Internacional sobre Promoção da Saúde em Ottawa - Canadá, em novembro de 1986; Segunda Conferência Internacional sobre a Promoção da Saúde, em Adelaide - Austrália, de 5 a 9 de abril de 1988; e a Terceira Conferência Internacional de Promoção da Saúde, realizada em Sundsvall - Suécia, no período de 9 a 15 de junho de 1991; Conferência Internacional de Promoção da Saúde em Santa Fé de Bogotá - Colômbia, em novembro de 1992; Quarta Conferência Internacional para a Promoção da Saúde, em Jacarta - Indonésia, em junho de 1997; Primeira Reunião Formal da Rede de Megapaíses, em Genebra - Suíça, em março de 1998; e a Quinta Conferência Internacional sobre a Promoção da Saúde, realizada na Cidade do México - México, em junho de 2000.

\section{OS EVENTOS INTERNACIONAIS SOBRE PROMOÇÃO DA SAÚDE: REFLEXÕES NA PERSPECTIVA DO CUIDADO À GESTANTE}

Os eventos internacionais de saúde desde a Conferência de Alma-Ata - Rússia, em setembro de 1978, ressaltam a importância de criar estratégias para melhorar a qualidade de vida da população como visualizava a meta: "Saúde para Todos no Ano 2000". Naquela Conferência, os cuidados primários de saúde eram as estratégias principais para se atingir essa meta, incluindo a Educação em Saúde no rol das atividades. Entre o elenco de atividades propostas incluiram-se os cuidados de saúde materno-infantil integrando-se neles, o planejamento familiar e a imunização. 0 uso de tecnologias simples, foi enfatizada como a Carta destaca: fazendo o mais pleno uso de recursos disponíveis, locais, nacionais e outros ao alcance da comunidade, valorizando a participação da sociedade 5:16.

A I Conferência Internacional sobre Promoção da Saúde, realizada em Ottawa - Canadá, em novembro de 1986, priorizou a saúde enfatizando-a como processo positivo, social e pessoal. 0 encontro de um bem-estar global tem como pré-requisito para a saúde condições e recursos fundamentais, tais como: paz; habitação; educação; alimentação; renda; ecossistema estável; recursos sustentáveis; justiça social e eqüidade ${ }^{5: 19}$

É importante ressaltar a reorientação dos serviços de saúde, descrita nas cartas, com ênfase na Carta de Ottawa da I Conferência Internacional sobre Promoção da Saúde, quanto ao cuidado, holismo e ecologia, temas considerados essenciais e indispensáveis na promoção da Educação em Saúde. 
Entre os importantes compromissos dessa Conferência, destacaram:

reconhecer as pessoas como principal recurso para a saúde; apoiá-las e capacitá-las para que se mantenham saudáveis à si próprias, às suas famílias e amigos, através de financiamento e/ou outras formas de apoio e aceitar a comunidade como porta voz essencial em matéria de saúde, condição de vida e bem-estar ${ }^{5: 23}$.

A II Conferência Internacional sobre Promoção da Saúde, realizada em Adelaide - Austrália, no ano de 1988, teve o tema "Políticas Saudáveis". A Conferência considerou as propostas da Carta de Ottawa tendo por desafio as mudanças relacionadas às novas políticas de saúde, que poderiam ser reais a partir do momento em que ocorresse uma conscientização global dos governos, organizações e pessoas ${ }^{5}$. Nesta Conferência ficou estabelecido que as mulheres são as principais promotoras da saúde no mundo. Referiu também, que todas as mulheres deveriam ter acesso à informaç̃̃es para que sua participação fosse efetiva. Reforçou as políticas públicas identificando quatro áreas e priorizando apoio à saúde da mulher, sendo estas:

Igualdade de direitos na divisão de trabalho existente; práticas de parto baseadas nas preferências e necessidades das mulheres; mecanismos de apoio à mulher trabalhadora como: apoio a mulheres com crianças, licença-maternidade, licença para acompanhamento dos cuidados a filhos doentes 5:28.

$\mathrm{Na}$ Conferência, a mulher ainda foi beneficiada ressaltandose sua liberdade de escolha e suas necessidades relacionadas com o parto. É oportuno ressaltar o apoio à mulher, referido na Declaração de Adelaide, que enfatiza os princípios naturais e fisiológicos, visando à satisfação do estado da parturiente. Também se destaca a finalidade preservar as preferências e direitos da população feminina para as usufruir a maternidade saudável e a assistência humanizada.

Essa Conferência ainda alertou a população quanto aos danos produzidos por tabagismo e abuso do álcool, que interferem negativamente durante a fase gestatória. Ressaltou a importância na criação de ambientes saudáveis, que protejam a saúde das pessoas conservando recursos naturais, usando estratégias ecológicas, local, regional e global. A referida Carta ainda recomenda novas propostas entre os diversos níveis de decisões e participantes. No tocante aos ambientes saudáveis, as políticas de saúde recomendam manter e conservar ambientes naturais de alcance global, nacional e local.

A III Conferência Internacional, em Sundsvall - Suécia, no ano de 1991, abordou o tema "Ambientes Favoráveis à Saúde". Fez um apelo a todos os povos habitantes de várias partes da Terra a se unirem com empenho, a fim de atuarem na promoção de ambientes favoráveis à saúde, visto que: ... milhões de pessoas vivem em situação de extrema pobreza e privação em ambientes degradado que ameaça cada vez mais a saúde ${ }^{5: 34}$. Reconheceu ainda que todas as pessoas têm o seu papel na criação de ambientes favoráveis à saúde, porquanto é prioridade básica a eqüidade para promoção da saúde. Reconheceu também, a educação como direito de todo cidadão com vistas a mudanças de ordens políticas, econômicas e sociais enfatizando a saúde à população.

Essa Conferência reforçou as propostas anteriores e acrescentou quatro estratégias:

ações comunitárias organizadas por mulheres, capacitar a comunidade na tomada de decisão e construir aliança nas áreas de saúde e ambiente favoráveis, mediar interesses conflitantes e assegurar o acesso igualitário 5:36.

As ações posśiveis para a criação de ambientes favoráveis à saúde têm diferentes dimensões: física, social, espiritual, econômica e política, estando intrinsecamente ligadas, numa interação dinâmica. Em resumo, os conferencistas reconheceram a educação como um direito humano básico sendo ela um elemento-chave nas mudanças políticas, econômicas e sociais, para tornar a saúde possivivel para todos, particularmente com relação à cultura, classe social e gênero.

A Conferência Internacional de Promoção da Saúde, em Santa Fé de Bogotá - Colômbia, em novembro de 1992, foi a versão latino-americana. Nela foram debatidas questões mais especíicas da região tendo como propósito fundamental, a saúde e o desenvolvimento. Enfatizou a situação epidemiológica caracterizada pelo ressurgimento de endemias, tais como malária, cólera, tuberculose e desnutrição. A permanência de outras doenças, tais como câncer e doenças cardiovasculares; o aparecimento da AIDS; e a degradação ambiental. Em razão dos agravantes provocados por essas doenças, fizeram-se maiores esclarecimentos à população, de modo geral, como estratégia para a promoção da saúde e particularmente, à mulher na fase reprodutiva da vida.

Em junho do ano de 1997, em Jacarta - Indonésia, realizou-se a IV Conferência Internacional para a Promoção da Saúde, a primeira realizada em um país em desenvolvimento. Ela inclui também o setor privado na promoção da saúde, para reexaminar os determinantes da saúde, na identiificação de novas estratégias que viabilizem uma melhor promoção da saúde futura, imprescindível ao desenvolvimento socioeconômico.

A Primeira Reunião Formal da Rede de Megapaíses, realizada em Genebra - Suíça, em março de 1998, foi baseada nas Conferências anteriores. Na Quinta Conferência Internacional sobre a Promoção da Saúde realizada na Cidade do México, em junho de 2000, reconheceu-se que a promoção da saúde e do 
desenvolvimento social é um dever e responsabilidade central dos governos, compartilhada por todos os setores da sociedade ${ }^{5: 53}$.

Concluiu-se nesta Conferência, que em todos os países, a promoção da saúde faz parte essencial e necessária das políticas e programas públicos, a fim de promover eqüidade e saúde para todos.

Nessa perspectiva, os programas de saúde requerem dos profissionais das diversas áreas, conhecimentos sobre as propostas referidas nas Cartas, com a finalidade de assegurar sua participação efetiva nas ações de promoção da saúde.

A preocupação com a saúde da gestante é ressaltada nas conferências (Quadro 1), destacando-se a assistência materno-infantil como proposta de cuidados para uma gravidez saudável, abrangendo o binômio mãe-filho. Nesses cuidados, estão incluídos a comunidade, o meio ambiente, a família e a educação.

Quadro 1

Síntese das propostas internacionais de atenção à saúde da mulher grávida. Fortaleza, 2004.

\begin{tabular}{|c|c|c|c|}
\hline Ano & Conferências" & Acontecimentos & $\begin{array}{c}\text { Recomendações } \\
\text { acerca da saúde } \\
\text { da mulher }\end{array}$ \\
\hline 1978 & Alma-Ata & & $\begin{array}{l}\text { - Educação e Saúde } \\
\text { - Saúde materno-infantil } \\
\text { - Planejamento familiar } \\
\text { - Tecnologia simples - recursos } \\
\text { disponíveis } \\
\text { - Participação da comunidade }\end{array}$ \\
\hline 1986 & Ottawa & & $\begin{array}{l}\text { - Educação } \\
\text { - Reorientação de serviços }\end{array}$ \\
\hline 1988 & Adelaide & & $\begin{array}{l}\text { - Políticas saudáveis - enfoque } \\
\text { e apoio às mulheres durante a } \\
\text { maternidade } \\
\text { - Práticas de parto, baseadas nas } \\
\text { preferências e necessidades das } \\
\text { mulheres. Cuidado com os } \\
\text { filhos doentes }\end{array}$ \\
\hline 1990 & $\begin{array}{l}\text { Conferência } \\
\text { Sanitária } \\
\text { Pan-Americana }\end{array}$ & & $\begin{array}{l}\text { - Vigilância da mortalidade } \\
\text { materna para a América Latina }\end{array}$ \\
\hline 1990 & & $\begin{array}{l}\text { Estatuto da criança e } \\
\text { do adolescente } \\
\text { Alojamento Conjunto } \\
\text { - obrigatório em to- } \\
\text { das as maternidades }\end{array}$ & $\begin{array}{l}\text { - Atendimento à gestante pré- } \\
\text { natal e parto pelo SUS } \\
\text { - Vínculo mãe/bebê } \\
\text { - Aleitamento materno }\end{array}$ \\
\hline 1991 & Sundsvall & & $\begin{array}{l}\text { - Movimento organizado } \\
\text { de mulheres }\end{array}$ \\
\hline
\end{tabular}

*As Conferências de Santa Fé (1992), Jacarta (1997), Genebra (1998) e Cidade do México (2000) foram comtempladas apenas no texto.

\section{AS AÇÕES SOBRE OS SUJEITOS - EDUCAÇÃO EM SAÚDE NA ATENÇÃO À GESTANTE}

0 conceito de educação em saúde tem sido entendido por alguns como a implementação de atividades educativas para induzir mudanças no indivíduo, especialmente no seu estilo de vida. Esse conceito é restrito e tem sido criticado, por ser estreito, com focalização individual, reprodutiva e reducionista 6 .

Assim, a educação em saúde passa a ser entendida como uma prática intencional e planejada, na qual os indivíduos possam ter domínio sobre o seu corpo através de aprendizagem contínua sobre a saúde e doença. Dessa forma, eles podem produzir mudanças na compreensão do pensar e fazer saúde, influenciar valores, introduzir mudanças nas crenças e atitudes, adquirir habilidades e reorientar as políticas públicas?.

Ao tratarmos da promoção da saúde-educação em saúde, estamos nos referindo aos aspectos que envolvem a relação entre sujeito e estrutura em uma relação dinâmica e interdependente que envolve a articulação entre saberes técnicos e populares e a mobilização de recursos institucionais, comunitários, públicos e privados ${ }^{8}$.

A educação abre janelas para um processo de transformação ${ }^{9}$. Desta maneira, a educação transformadora é importante para reforçar a promoção da saúde e as estratégias propostas nas conferências abordadas.

A educação em saúde, como prática social do enfermeiro/enfermeira, deve partir do entendimento de que a realidade de saúde pode fazer muito por si mesma, desde que conte com os conhecimentos, a compreensão e a motivação necessárias para refletir sobre ela.

Torna-se fundamental que os profissionais da saúde envolvidos com o processo entendam as propostas das Cartas e trabalhem neste desafio, no sentido de contribuir para a promoção da saúde para que todos os seres do Planeta possam usufruir de uma vida saudável. Este pensamento corrobora-se plenamente com o papel fundamental do profissional de saúde no processo educativo para a promoção da saúde coletiva e à mulher ${ }^{10}$. Portanto, o profissional, zeloso e consciente de suas responsabilidades no cuidado para a gestante, deve ser mais um educador na promoção da saúde lembrando que, de acordo com a Constituição Federal de 1988, artigo 196, a saúde é direito de todos e dever do Estado ${ }^{11}$.

A educação, é um dos principais componentes no cuidado com a gestante, a parturiente, puérpera e família 12:104. Entenda-se este cuidado, no plano ambulatorial, hospitalar ou domiciliar proporcionando oportunidade aos profissionais da saúde de fazerem uma reflexão crítica em relação aos aspectos que interferem na saúde das gestantes e, assim, tentar superá-los criando estratégias para que elas 
próprias, com o reforço do profissional, adotem as medidas de promoção da saúde e prevenção de doenças.

A educação e a saúde estão intimamente ligadas ao exercício da cidadania, aos direitos e deveres do cidadão, na luta por melhores condições de vida ${ }^{13}$. Torna-se necessário estabelecer interação enfermeiro/cliente para que a ação educativa possa ajudar a mulher na compreensão e conhecimento do seu corpo, gravidez, sintomas do trabalho de parto, exercícios e do alívio da dor do parto e manutenção da respiração oxigenadora durante as contrações, entre outros aspectos. Mediante 0 entendimento desses conhecimentos, ela pode participar do cuidado contribuindo para diminuição da angústia e da tensão que envolve esse processo. Nesse sentido, o envolvimento diário do enfermeiro com a clientela, durante o pré-natal e por todo o trabalho de parto e parto, torna-se imprescindível.

0 cotidiano da assistência de enfermagem é permeado por situações que necessitam uma atuação voltada para a Educação em Saúde ${ }^{14}$ Nesse sentido, é necessário que o(a) enfermeiro(a) use de estratégias, tais como: formação de grupos de gestantes, nos quais elas possam participar de atividades de recreação, lazer e relaxamento; expressar suas necessidades, queixas e dúvidas; trocar idéias; contar as suas experiências anteriores sobre o parto; e receber orientação sobre exercícios apropriados para este período. Essas estratégias devem ser adequadas, ajustadas às necessidades da grávida, e requerem atitudes transformadoras por parte dos envolvidos a fim de contribuir para o desenvolvimento saudável da gravidez.

Tais conhecimentos deverão ser iniciados a partir das consultas de enfermagem pré-natal, dada a importância de se estabelecer uma aproximação enfermeiro(a)-cliente, com a finalidade de dar esclarecimentos às gestantes sobre dúvidas bastante comuns no período da gravidez e, dessa forma, torná-la conhecedora de como ocorrem as mudanças corporais na gravidez, o trabalho de parto e parto e ter informações acerca da higiene, alimentação e ambientação consideradas indispensáveis, em se tratando de cuidados de enfermagem e de promoção de saúde à grávida.

0 acolhimento tem o significado de ouvir, acolher e dar atenção. É um dos objetivos do pré-natal. Isto porque, a gestante necessita de ser ouvida. As queixas da gestante podem representar para ela um problema que precisa ser esclarecido. Daí, a necessidade de esclarecimentos seguros, tão significativos para o seu bem-estar e de sua familia4. Acolher é para o MS, um processo de inclusão do usuário do senviço de saúde, na rede integrada de atendimento multiprofissional ${ }^{15}$.

Faz-se necessário que, durante as consultas de enfermagem, a enfermeira faça um aconselhamento, que é definido como:
Um processo de escuta ativa, individualizado e centrado no cliente. Pressupõe a capacidade de estabelecer uma relação de confiança entre os interlocutores, visando ao resgate dos recursos internos do cliente para que ele mesmo tenha a possibilidade de reconhecer-se como sujeito de sua própria saúde e transformaçãa ${ }^{16: 9}$

0 aconselhamento abrange: a importância do prénatal, direitos e deveres da gestante, informações sobre a prevenção das DSTS/AIDS, outras doenças que acometem as mulheres grávidas e os malefícios e conseqüências que o tabaco produz no desenvolvimento fetal, tais como, descolamento prematuro da placenta, aborto, baixo peso ao nascer, entre outros.

Assim, a adequação de informações, no sentido de prevenir possíveis complicações, é um passo primordial que deve ser discutido e reforçado com a gestante, em todas as consultas pré-natais subseqüentes.

0 Ministério da Saúde ressalta que a ação educativa realizada através de metodologias participativas enseja a possibilidade de intercâmbio de experiências e conhecimentos sendo considerada a melhor forma de promover a compreensão do processo de gestação e parto. Entre as diferentes formas de realização do trabalho educativo, destacam-se as discussões em grupo, as dramatizações e outras dinâmicas que facilitam a fala e a troca entre os componentes do grupo ${ }^{17}$.

\section{SOBRE AS AÇÕES ESTRUTURAIS DAS POLÍTICAS DE PROMOÇÃO À SAÚDE DA MULHER}

No Ceará, nos anos de 1975 a 1985, o professor e médico Galba Araújo deu início às primeiras casas de parto, aproveitando ambientes naturais, respeitando os costumes regionais, as condições locais, contando com parteiras conhecidas e de confiança da população, em localidades interioranas e zonas de praia. Essas casas de parto eram conduzidas por parteiras leigas. 0 projeto teve 0 apoio da Universidade Federal do Ceará e da Maternidade Escola Assis Chateaubriand.

No entanto, as maternidades no Brasil estão iniciando agora, embora ainda timidamente, o proposto na Carta de Adelaide, isto é, as práticas de parto baseadas nas preferências das mulheres ${ }^{5: 28}$ Para tal acontecimento, é importante que haja necessidade de promover, no ambiente hospitalar, o respeito pelo processo do parto e nascimento, acatando a individualidade humana e a abordagem interdisciplinar como um todo.

Nessa óptica, insere-se no exercício da prática profissional um novo conceito de saúde que considere a consciência plena de cidadania dos indivíduos exige novas posições e, portanto, novos ordenamentos culturais 18:16

Em Fortaleza, CE, no ano de 1985, a Organizaçã̃o Pan-americana de Saúde e a Organização Mundial da 
Saúde promoveram a Conferência sobre a Tecnologia Apropriada para o Nascimento, com recomendações, informações e conhecimentos sobre a prática da assistência ao parto normal ${ }^{10}$.

As recomendações da Conferência de Adelaide foram observadas no campo de atuação da Enfermagem Obstétrica. Também as políticas públicas foram elaboradas para assegurar a qualidade de atenção à mulher grávida. Essas orientações e mais os requisitos contidos nas Cartas sobre práticas de parto foram incorporadas na Portaria 985/99, do Ministério da Saúde do Brasil, quando da criação das casas de parto em agosto de 1999. Com o nome de Centro de Parto Normal, adota o modelo humanizado de assistência ao parto sem distocia e assistência ao parto realizado por enfermeira obstetra. Também valoriza a mulher e sua preferência quanto ao modo de parir, recebendo acompanhamento do companheiro ou pessoa de sua livre escolha. Esses requisitos estão sendo contemplados nos centros de parto normal (casas de parto) de algumas cidades brasileiras.

Atendendo a proposta apresentada nas Conferências Internacionais de Adelaide e Sundsvall, as iniciativas estão sendo postas em funcionamento em alguns locais, destacando-se: Casa de Parto Sapopemba, SP, gerenciada por enfermeira obstetra; Casa de Parto Dr. David Capistrano, em Realengo, Rio de Janeiro, gerenciada também por uma enfermeira obstetra, entre outras existentes no país.

As casas de parto, onde 0 ambiente assemelha-se ao doméstico, apresentam-se agradáveis e aconchegantes, além de refletirem bem-estar. São locais apropriados para a realização do parto normal, no qual crenças e valores são respeitados e as pessoas podem desenvolver a auto-estima. 0 companheiro pode acompanhar e assistir ao parto. Entende-se que observações destes cuidados respondem às recomendações de favorecimento de um ambiente saudável e apropriado para assistir o parto e o nascimento.

Ressalta-se que, nessas casas de parto, a realização do parto normal sem distocia por enfermeira obstetra é uma realidade, em consonância com a legislação em vigor: Lei n.7498/86 e Decreto Lei 94406/ 87 (que regulamenta a Lei), bem como Portarias do Ministério da Saúde 2815/98 e 163/98 e Resolução do COFEn 223/99, que dispõe sobre a atuação de enfermeiros na assistência à mulher no ciclo gravidicopuerperal, considerando que a Portaria $n^{\circ}$ 63/22/98 (MS), publicada no DOU $n^{0} 183$, regulamenta a realização do procedimento parto normal realizado por enfermeiro obstetra'.
Em 13 de julho de 1990, foi aprovado o Estatuto da Criança e do Adolescente, Lei Federal $n^{0} 8069$, que assegura à gestante adolescente 0 atendimento prénatal e o parto pelo Sistema Único de Saúde. Também trata da obrigatoriedade do alojamento conjunto ${ }^{10}$.

Ainda como resposta à demanda dessa nova reorientação dos serviços, com o apoio do Ministério da Saúde, um conjunto de medidas foi proposto considerando a importância da atenção obstétrica e perinatal e a humanização dos serviços, entre eles: Projeto Canguru, em junho de 1994, lançado no Instituto MaternoInfantil de Pernambuco (IMIPI) recebendo o Prêmio Galba Araújo (em 1996), criação das Casas de Parto (Portaria 985/GM, de 05 /08/1999) e financiamento de Cursos de Especialização em Enfermagem Obstétrica considerando os critérios determinados pela Associação Brasileira de Obstetrizes e Enfermeiros Obstetras ${ }^{20}$; Programa de Humanização do Atendimento ao Pré-natal e Nascimento (PHPN), através da Portaria do Ministério de $n^{0} 569$ de 1 de junho de 2000, que estabelece realização da primeira consulta à gestante até 0 quarto mês de gestação e no mínimo seis consultas pré-natal, entre outros procedimentos ${ }^{4}$.

Em junho do ano 2000, o Ministério da Saúde elaborou manuais sobre 0 atendimento à saúde materno-infantil, dando ênfase à humanização do parto e do nascimento. Destacam-se as Portarias n569 e n ${ }^{\circ}$ 570, de 01 de junho de 2000, que instituem o Programa de Humanização no Pré-natal e Nascimento ${ }^{18}$.

Outras organizações estrangeiras, como o Fundo das Nações Unidas para a Infância (UNICEF), deram apoio para a redução da mortalidade materna. Em Fortaleza, no ano de 1996, a Agência de Cooperação Internacional do Japão (JICA) em parceria como o Ministério da Saúde e a Secretaria de Saúde do Estado do Ceará e o Projeto Luz desenvolveram, no Estado do Ceará, programas com objetivos de: expandir ações sobre a maternidade segura, à luz da humanização do parto e nascimento; proporcionar bem-estar para a parturiente e reduzir os riscos de saúde para o bebê; melhorar 0 apoio às mulheres durante o pré-parto e pós-parto, com a meta específica de melhorar a saúde materna e neonatal; além de realizar treinamentos de médicos e enfermeiros no contexto local, nacional e internacional, com os objetivos de humanizar o parto.

De acordo com a Portaria 2104/GM, em 19/11/ 2002, foi instituído o Projeto Nascer, com o objetivo de evitar a transmissão vertical de AIDS, aumentar a cobertura do tratamento da AIDS e da sífilis nas maternidades brasileiras, com a realização do teste rápido anti-HIV e do VDRL ${ }^{15}$. 
Estudos ilustram a situação atual em que a mulher está sendo cuidada durante o trabalho de parto e parto ${ }^{10: 21}$. Refletimos, sobremodo, na qualidade de atendimento à mulher, do que está acontecendo ao seu redor, sobre os procedimentos recebidos desde o pré-natal.

Nesse sentido, tornou-se prioridade, segundo o Ministério da Saúde, considerar, nesse processo, os desejos e valores da mulher e adotar uma postura sensível e ética, respeitando-a como cidadã e eliminando as violências verbais e não verbais ${ }^{18}$. A postura ética deve ser implícita entre todos os profissionais da saúde, isto é, zelar pela mulher e respeita-la, quer durante o prénatal, o parto ou durante o dia-a-dia do profissional.

A qualidade da assistência obstétrica na comunidade tem demonstrado que ainda falta muito para se atingir o que foi preconizado a partir das conferências internacionais.

\section{CONSIDERAÇÕES FINAIS}

Com o presente estudo, fez-se uma reflexão sobre a promoção da saúde à gestante, ressaltando as recomendações presentes nos eventos internacionais de promoção da saúde. Foi possível perceber a importância das propostas nelas contidas para a melhoria da saúde dos habitantes do Planeta, mesmo que a meta Saúde para todos no ano 2000 não tenha sido atingida.

\section{Referências}

1. Ministério da Saúde (BR). RIPSA. Rede Interagencial de Informações para a saúde [on-line200[citado10 ago 2005] Disponível em: http:/ /www.portal.saude.gov.br.htm.

2. Costa AAR, Ribas SSS, Amorim MMR, Santos CL. Mortalidade Materna na cidade de Recife. Rev Brasileira Ginecologia e Obstetrícia. [online] 2002 [citado 12 out 2005] Disponível em : http:// www.portal.mortalidadematerna.gov.br.

3. Rede Feminista de Saúde. Em cada cidade um comitê de prevenção da mortalidade materna: dever do Estado, compromisso da sociedade. [on-line]2003 [citado 12 jun 2003] Disponível em: http www.redesaude.org.br.htm.

4..Ministério da Saúde (BR). Secretaria da Saúde. Saúde Reprodutiva e Sexual: um manual para a atenção primária e secundária (nivelo ambulatorial). Fortaleza (CE): Secretaria da Saúde do Ceará ;2002.
Destaca-se a importância do papel do (a) enfermeiro (a) como educador (a), na promoção da saúde e prevenção de doenças, como facilitador de mudanças na comunidade e na gestante, quanto ao saber cuidar-se durante o processo gravídico e puerperal.

Em todas as Cartas, há um reforço para a promoção da saúde, suas prioridades no sentido de conduzir um comportamento positivo dentro das políticas de saúde e de programas públicos.

É necessário retomar reflexões sobre a ação educativa à gestante contida nas Cartas sobre Promoção da Saúde, a partir das políticas públicas relacionadas à saúde da mulher, bem como à saúde reprodutiva de um modo geral.

A complexidade do processo saúde-doença continua presente no mundo como agravante junto às populações carentes. Contudo, a qualidade da assistência obstétrica na comunidade tem demonstrado que, mesmo com os esforços e a ampliação dos serviços de saúde à população, ainda há muito a percorrer para se atingir o que foi preconizado pelas Conferências.

Dessa forma, cabe repensar a seguinte afirmação: articular responsabilidade, liberdade e compromisso significa que, ao educador ou ao profissional de saúde, não basta saber. É preciso também querer e não adianta saber e querer, se não se tem poder para acionar os mecanismos de transformação nos rumos da instituição que se quer mudar - os serviços de saúde ${ }^{21: 75}$.

5. Ministério da Saúde (BR). Secretaria de Políticas de Saúde. Projeto Promoção da Saúde. Promoção da saúde: declaração de Alma-Ata, Carta de Ottawa, Declaração de Sundswal, Declaração de Adelaide, Declaração de Jacarta, Declaração de Santa Fé de Bogotá, Rede de Mega Países, Declaração do México. Brasília (DF): 2001.

6. Macdonald T. Health Promotion : Disciplines and Diversity. London: Routledge,1998.

7. Tones K.Health Education as empowerment. Houndsmills: MacMillam Press, 1997.

8. Buss PM. Uma introdução ao conceito de promoção de saúde. In: Promoção da saúde: conceitos, reflexões, tendências. Rio de Janeiro (RJ): Fiocruz, 2003.

9. Freire P. Educação e mudança. 23ª ed. São Paulo: Paz e Terra; 1999.

10. Bessa LF. Ação educativa: uma perspectiva para a humanização do parto? [tese doutorado]. Ribeirão Preto: Escola de Enfermagem da Universidade de São Paulo; 2002. 
11. Brasil. Constituição: 1988. Brasília (DF): 2003.

12. Oliveira ME, Zampieri MFM, Brüggemann OM. Interdisciplinaridade o som da orquestra. In: A melodia da humanização: reflexões sobre o cuidado no processo do nascimento. Florianópolis: Cidade Futura; 2001.

13. Costa ICC, Fuschela MAP. Educação e saúde: importância da integração dessa prática na simplificação do saber. Rev Ação Coletiva, 1999.

14. Wall ML. Tecnologias educativas: subsídios para a assistência de enfermagem a grupos Goiânia: AB; 2001.

15.Ministério da Saúde (BR). Secretaria Executiva. Programa Nacional DST e AIDS. Projeto Nascer. Brasília (DF); 2001.

16. Ministério da Saúde (BR). Secretaria de Políticas de Saúde. Coordenação Nacional de DST e AIDS. Aconselhamento: um desafio para a prática integral em saúde. Brasília (DF); 1999.

17. Ministério da Saúde (BR). Secretaria de Políticas de Saúde. Área Técnica da Saúde da Mulher. Assistência pré-natal: manual técnico. Brasília (DF); 2000.

18. Ministério da Saúde (BR). Secretaria de Políticas de Saúde. Área Técnica da Saúde da Mulher. Parto, aborto e puerpério: assistência humanizada à mulher. Brasilia (DF); 2001.

19. Pereira VM, Maranhão MASA, Andreoni S. Dispositivos legais relacionados à saúde da mulher e do recém-nascido: manual. São Paulo: Associação Brasileira de Enfermeiros Obstétricos; 2002.

20. Sacramento MTP, Tyrrell MAR. Perspectiva da avaliação educacional nos cursos de especialização em Enfermagem Obstétrica. Esc Anna Nery Rev Enferm. 2004 ago.; 8 (2): 286-90.

21. Moura MAV, Wolf LR. A institucionalização do parto e a humanização da assistência: revisão de literatura. Esc Anna Nery Rev Enferm. 2004 ago.; 8 (2): 279-85.

\section{Sobre as Autoras}

Isolda Pereira da Silveira

Enfermeira Obstetra da Maternidade Escola Assis Chateaubriand-MEAC/ UFC. Mestre em Enfermagem.

E-mail: isolda_silveira@yahoo.com.br

Antônia do Carmo Soares Campos

Enfermeira da UTI - Neonatal da MEAC/UFC. Doutoranda em Enfermagem/UFC. Docente do Curso de Graduação em Enfermagem da Universidade de Fortaleza-UNIFOR.

E-mail: ankardagostinho@terra.com.br.

\section{Francisca Ana Martins Carvalho}

Enfermeira. Doutoranda em Enfermagem/UFC. Professora do Departamento de Enfermagem da Faculdade de Farmácia, Odontologia e Enfermagem, da Universidade Federal do Ceará-DENF/FFOE/UFC.

\section{Maria Grasiela Teixeira Barroso}

Enfermeira, Livre Docente, Professora do DENF/FFOE/UFC.

E-mail: grasiela@ufc.br. Livre

\section{Neiva Francinely Cunha Vieira}

Enfermeira, Doutora e Professora do DENF/FFOE/UFC.

E-mail: nvieira@ufc.br. 